\title{
Monocular patching in subjects with right-hemisphere stroke affects perceptual-attentional bias
}

\author{
Anna M. Barrett, MD; ${ }^{1-2 *}$ Stephanie Burkholder, BA ${ }^{3}$ \\ ${ }^{1}$ Kessler Medical Rehabilitation Research and Education Corporation, West Orange, $N J ;{ }^{2}$ Department of Physical \\ Medicine and Rehabilitation, University of Medicine and Dentistry of New Jersey, Newark, NJ; ${ }^{3}$ Department of Neurol- \\ ogy, Pennsylvania State University College of Medicine, Hershey, PA
}

\begin{abstract}
Monocular patching is a possible inexpensive treatment for spatial neglect. Previous studies were unpromising, but since neglect symptoms are heterogeneous, fractionating spatial bias may detect significant effects of patching. Poststroke, perceptual-attentional (PA) spatial bias, motor-intentional (MI) spatial bias, or both may occur. In this study, six poststroke subjects bisected lines while self-monitoring their performance via a camera/video apparatus. We dissociated PA and MI spatial bias by right-left reversing visual feedback in some trials. Subjects were tested with and without right and left eye patches. Patching did not affect group line-bisection error, but both right and left patches decreased individual subject PA spatial bias $(p<0.05)$. We detected no patching effect on individual subject MI spatial bias (significant patch side by bias interaction, $p=$ 0.03 ). When we examined each subject's results separately, patching improved performance in subjects who had greater PA and MI spatial biases. We conclude that monocular patching may primarily affect poststroke PA spatial bias. Further studies on this intervention are needed.
\end{abstract}

Key words: attention, cognition, eye patch, monocular vision, motor planning, neglect, rehabilitation, spatial bias, stroke, visual occlusion.

\section{INTRODUCTION}

Spatial neglect has been defined as a person's failure to report, respond, or orient to a novel or meaningful stimulus in the side of space contralateral to a brain lesion [1]. It commonly occurs after a right-hemisphere stroke and is a robust predictor of poststroke loss of independence [2]. Effective treatments for this condition are needed; although a number of treatments are reported, an approach that consistently results in significant improvement is not yet available [3]. Satisfactory spatialneglect treatment may require specific therapies directed at the neuropsychological mechanisms of the deficit, which may differ in individual patients [4].

A potential obstacle to effective clinical treatment studies on spatial neglect is poor clinical agreement on appropriate study inclusion criteria. Different studies included subjects with markedly different symptom profiles, and some studies that have been cited as relevant to spatial-neglect treatment included chronic patients with spatial bias who might not have been functionally impaired by their deficit [5]. A proposed revised definition for spatial neglect syndrome that may reduce this confusion is "spatial bias in a subject with a brain lesion, associated with functional disability." This definition is consistent with the traditional primary emphasis on how

Abbreviations: $\mathrm{BIT}=$ Behavioral Inattention Test, $\mathrm{MI}=$ motor-intentional, $\mathrm{PA}=$ perceptual-attentional, $\mathrm{RL}=$ right-left (reversed).

*Address all correspondence to Anna M. Barrett, MD; Kessler Medical Rehabilitation Research and Education Corporation, 1199 Pleasant Valley Way, West Orange, NJ 07052; 973-243-6978; fax: 973-243-6984.

Email: abarrett@kmrrec.org

DOI: 10.1682/JRRD.2005.01.0015 
illness alters function, which is common to all therapeutic disciplines.

Sprague performed classic experiments on unilateral inattention in cats and demonstrated that the superior colliculus plays an important role in orienting behaviors [6]. When cats with unilateral cortical lesions and contralesional orienting failure received surgical ablation of the contralateral superior colliculus, their orienting deficit improved. An extremely simplified explanation of this "Sprague effect" is to posit that ablation of the contralateral superior colliculus disinhibited the ipsilesional colliculus and improved orienting contralesional to the cortical injury.

Posner and Rafal made the interesting suggestion, however, that this research might be relevant to people with spatial neglect [7]. Because the colliculi, unlike the cortex, have heavily monocular input, Posner and Rafal suggested that humans with left spatial neglect might recover contralesional orienting while wearing a right eye patch, thus decreasing input to the left colliculus. Another possible benefit of right eye patching, or blocking vision on the right in some other fashion, in subjects with spatial neglect is inducement of leftward orienting by limiting stimuli presented for response, which is analogous to con- straint-induced movement therapy [8]. Researchers subsequently reported that right eye patching, or hemipatching of the right visual field [9], reduces neglect in some right hemisphere-damaged patients [10-13]. However, we actually observed that ipsilesional eye patching was associated with ipsilesional errors that cannot be explained by the Sprague effect [14]. Individual responses in other subsequent studies have been mixed (Table 1).

In previous studies, we observed that monocular patching primarily affected perceptual-attentional (PA) rather than motor-intentional (MI) spatial bias [14-15]. That eye patching primarily affects PA spatial systems seems straightforward if one views monocular patching as selective sensory deprivation. However, this treatment may also have a primarily MI effect. Sprague reported that collicular ablation improved orienting behaviors to food and response to threat in some animals with contralesional impairment after cortical lesions. However, he reported that collicular ablation in other animals resulted in marked ipsilesional circling even in the absence of a stimulus that could be classified as a pure motor bias. Wallace et al. hypothesized that the Sprague effect itself may be mediated via induced asymmetric striatal-motor

Table 1.

Effect of eye patching treatments on visual attention in individual poststroke patients: Summary of studies.

\begin{tabular}{|c|c|c|c|}
\hline Effect & Ipsilesional Eye Patching & Contralesional Eye Patching & Other \\
\hline Benefit & $\begin{array}{l}\text { Serfaty et al., } 1995[1]^{*} \\
\text { Soroker et al., } 1994[2]^{*} \\
\text { Butter and Kirsch, } 1992[3]^{*} \\
\text { Walker et al., } 1996[4]^{*}\end{array}$ & $\begin{array}{l}\text { Serfaty et al., } 1995[1]^{\dagger} \\
\text { Barrett et al., } 2001[5]^{\dagger}\end{array}$ & $\begin{array}{l}\text { Beis et al., } 1999 \text { [6]: Ipsile- } \\
\text { sional hemipatch, both eyes }\end{array}$ \\
\hline Worsening & $\begin{array}{l}\text { Soroker et al., } 1994[2]^{\dagger} \\
\text { Barrett et al., } 2001[5]^{\dagger} \\
\text { Walker et al., } 1996[4]^{\dagger}\end{array}$ & $\begin{array}{l}\text { Serfaty et al., } 1995[1]^{*} \\
\text { Soroker et al., } 1994[2]^{*} \\
\text { Barrett et al., } 2004[7]^{*}\end{array}$ & - \\
\hline No Effect & $\begin{array}{l}\text { Beis et al., } 1999 \text { [6] } \\
\text { Barrett et al., } 2004 \text { [7] } \\
\text { Walker et al., } 1996[4]\end{array}$ & - & - \\
\hline \multirow{2}{*}{\multicolumn{4}{|c|}{$\begin{array}{l}\text { 1. Serfaty C, Soroker N, Glicksohn J, Sepkuti J, Myslobodsky MS. Does monocular viewing improve target detection in hemispatial neglect? Rest Neurol Neuro- } \\
\text { sci. 1995;9:77-83. } \\
\text { 2. Soroker N, Cohen T, Baratz C, Glicksohn J. Is there a place for ipsilesional eye patching in neglect rehabilitation? Behav Neurol. 1994;7:159-64. } \\
\text { 3. Butter CM, Kirsch NL. Combined and separate effects of eye patching and visual stimulation on unilateral neglect following stroke. Arch Phys Med Rehabil. } \\
\text { 1992;73(12):1133-39. [PMID: 1463376] }\end{array}$}} \\
\hline & & & \\
\hline \multicolumn{4}{|c|}{$\begin{array}{l}\text { 4. Walker R, Young AW, Lincoln NB. Eye patching and the rehabilitation of visual neglect. Neuropsychol Rehabil. 1996;6(3):219-32. } \\
\text { 5. Barrett AM, Crucian GP, Beversdorf DQ, Heilman KM. Monocular patching may worsen sensory-attentional neglect: a case report. Arch Phys Med Rehabil. } \\
\text { 2001;82(4):516-18. [PMID: 11295013] }\end{array}$} \\
\hline \multicolumn{4}{|c|}{$\begin{array}{l}\text { 6. Beis JM, Andre JM, Baumgarten A, Challier B. Eye patching in unilateral spatial neglect: efficacy of two methods. Arch Phys Med Rehabil. 1999;80(1):71-76. } \\
\text { [PMID: 9915375] }\end{array}$} \\
\hline \multicolumn{4}{|c|}{$\begin{array}{l}\text { 7. Barrett AM, Crucian GP, Heilman KM. Eye patching biases spatial attention after thalamic hemorrhage in a patient without spatial neglect: a case study. Arch } \\
\text { Phys Med Rehabil. 2004;85(6):1017-20. [PMID: 15179660] }\end{array}$} \\
\hline \multicolumn{4}{|c|}{ *Theoretically expected effect. } \\
\hline
\end{tabular}


activity [16]. This is of pragmatic importance in rehabilitation. Eye patches are commonly used for ocular indications in poststroke patients undergoing rehabilitation [17], many of whom may have attentional problems. If eye patches can induce spatial neglect and associated functional deficit [15], patients' neurological recovery may be inadvertently impaired.

We discussed earlier the need for more developed inclusion and exclusion criteria for studies of poststroke spatial-neglect treatment. To move toward systematic clinical trials in this area, we need better information about which patients who have or have had spatial neglect should be included in a given study. Until more is known, case studies and case series reports will be invaluable sources of qualitative information about treatments.

In this study, we examined the possible effect of monocular patching on people in the chronic phase of stroke recovery. Even a small beneficial effect on this group, for whom few other attentional treatments are available, may be noteworthy. Although the study subjects did not demonstrate pathological spatial bias of a magnitude conventionally used to define neglect, they were selected because they demonstrated spatial bias and associated functional disability that met the revised spatial-neglect definition that we suggested earlier.

We aimed to assess whether eye patching in postright hemisphere stroke patients would primarily affect the PA or MI spatial systems. However, this issue can be addressed by analyzing subject performance in two ways. First, in previous studies we assumed that, across individuals, patching might produce a change in PA or MI spatial bias $[14,18]$. We assumed that, in each subject, both PA and MI spatial bias may contribute to possible performance errors. A specific subject performing a specific task may demonstrate not only a large magnitude (primary) PA-bias component for that task [19] but also a smaller magnitude MI-bias component. Monocular patching might, for example, affect PA spatial bias across individuals. However, if a specific subject's PA spatial bias does not account for the largest proportion of his or her performance errors, an eye patch might actually increase errors and change his or her performance from normal to abnormal.

A second approach assumes that we might see different effects of patching in groups of subjects who are categorized by their largest-magnitude (primary) spatial bias. Subjects whose primary spatial bias was one type (e.g., PA) might thus exhibit large magnitude monocular patching effects, while subjects whose primary spatial bias was another type (e.g., MI) might not show any effects. A difficulty with this approach is that methods for subtyping patients by spatial bias appear to be task-specific [20].

Because we did not know whether analyzing our data across subjects or within categorically defined subject groups was more appropriate, we carried out a pilot study in a small group of subjects. We analyzed the effect of monocular patching on PA and MI spatial biases in the study group as a whole and in individual subjects. We hoped, thereby, to refine criteria for outcome analysis in future, larger-scale studies of this intervention.

\section{METHODS}

\section{Subjects}

We tested six right-handed subjects (two men, four women, mean age 67.2 years, range 48-79 years) who were referred to our research project upon their discharge from a hospital neurological rehabilitation unit. Research referral occurred for post right-hemisphere stroke patients who were diagnosed with spatial neglect by their neurologist and/or occupational therapist in the acute stroke period; however, subjects took part in actual testing a mean 11.3 months poststroke (range 4-36 months). According to clinical notes, two subjects (4 and 6) had visual field defects as a result of their stroke: subject 4 had a left inferior quadrantanopsia and subject 6 a left homonymous hemianopia. Perimetry was unfortunately not performed on any subject in this study. Subject 4 was noted to have acute "left-sided grasp on line bisection" despite right gaze preference and left-sided omission errors on spatial testing. Subject 6 made left-sided omission errors on a line-cancelation array at the time of this study, which suggested chronic spatial neglect.

In this study, five of the poststroke subjects were tested with the Behavioral Inattention Test (BIT) [21] and scored at or above the recommended pathological cutoff score (mean 138, range 129-144). The BIT was mistakenly omitted from the testing protocol of subject 6 who was 36 months poststroke. Although we did not collect detailed data on the subjects' ability to perform activities of daily living, none of the subjects was living independently at the time of testing. Pertinent demographic and clinical information are shown in Table 2.

We also tested eight healthy controls with no history of neurological, psychiatric, or major medical illness (four women, mean age 73.6 years, range 69-86 years). 
JRRD, Volume 43, Number 3, 2006

Table 2.

Demographic and clinical characteristics of poststroke subjects in current study.

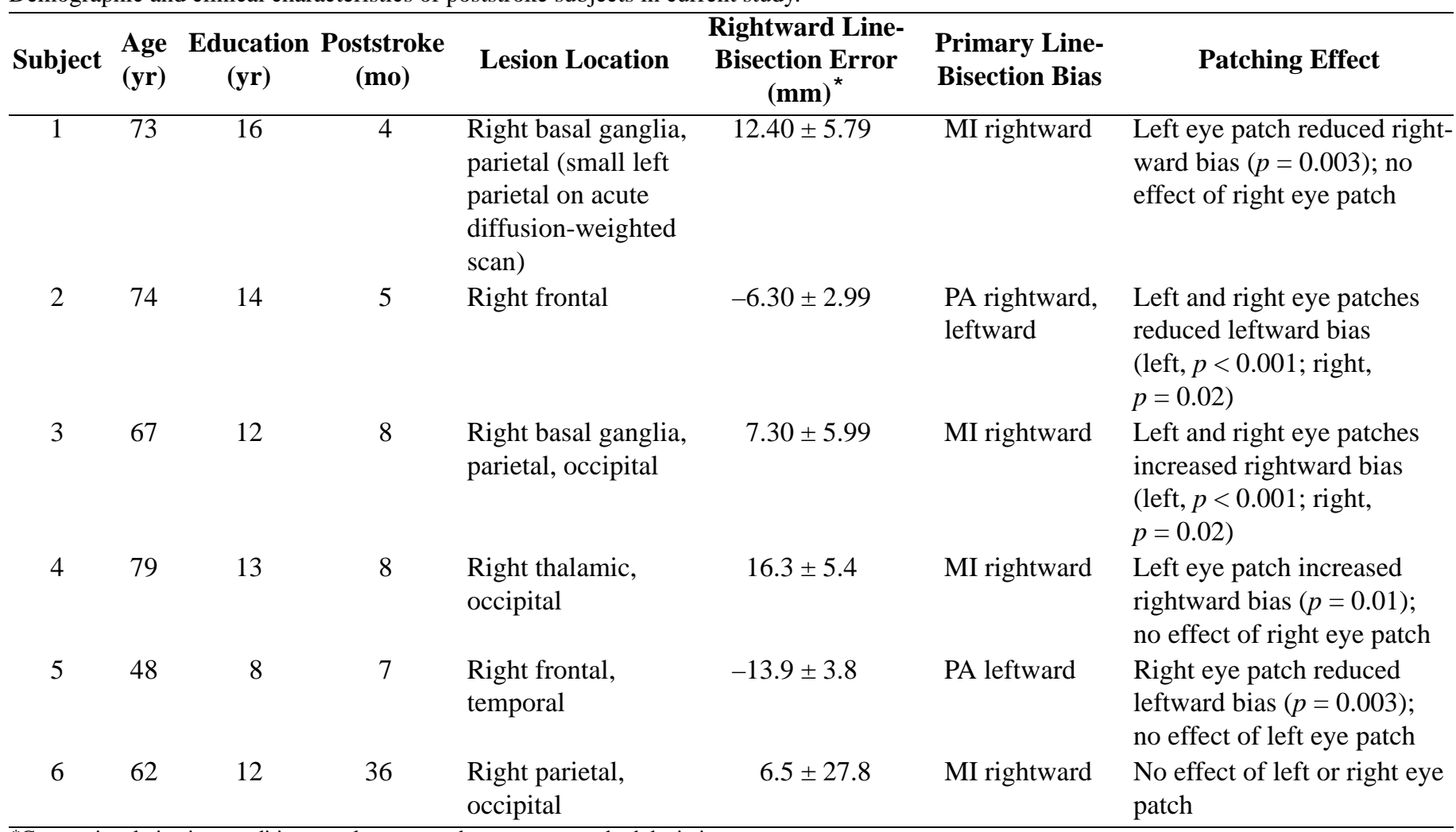

${ }^{*}$ Conventional viewing condition; results presented as mean \pm standard deviation.

$\mathrm{MI}=$ motor-intentional, $\mathrm{PA}$ = perceptual-attentional.

\section{Apparatus/Procedure}

\section{Calculation of Perceptual-Attentional and Motor- Intentional Components of Spatial Bias}

Subjects performed a $24 \mathrm{~cm}$ line-bisection task under two video-monitoring conditions (Figure 1). The apparatus has been described previously [4]. Briefly, subjects marked the center of a line on a piece of paper that was in a work space on a table in front of them. However, rather than directly viewing their hand and the line to be bisected, subjects looked at an eye-level video screen on which the line, paper, and their hand were projected via a video camera trained on the work space. Eye and head movements were unrestrained but observed while the subjects performed the line-bisection task. None of the subjects adopted an abnormal head or neck posture.

Subjects marked the center of the line under two conditions: (1) a conventional (congruent) condition, in which what they saw on the video screen corresponded to the left and right sides of the actual work space; and (2) a reversed (indirect) condition, in which what they saw on the video screen was right-left (RL) reversed from the actual work space. In the RL-reversed condition, rightward movement of a subject's hand in the work space appeared leftward on the screen and leftward movement appeared rightward. In this study, subjects always performed the RL-reversed condition after the conventional condition.

We algebraically determined the contribution of PA and MI spatial bias components to a subject's linebisection bias. A fraction of line-bisection error attributable to MI errors (failure to move leftward) versus PA errors (unawareness of the left) can be calculated if one assumes that under conventional conditions

$$
\text { Total Error = MI + PA Error. }
$$

By RL reversing what is viewed in the work space, one reverses the direction of PA feedback but not the direction of MI output. Thus, total error in the RLreversed condition can be expressed by the formula

$$
\text { Total Error RL Reversed = MI - PA, }
$$




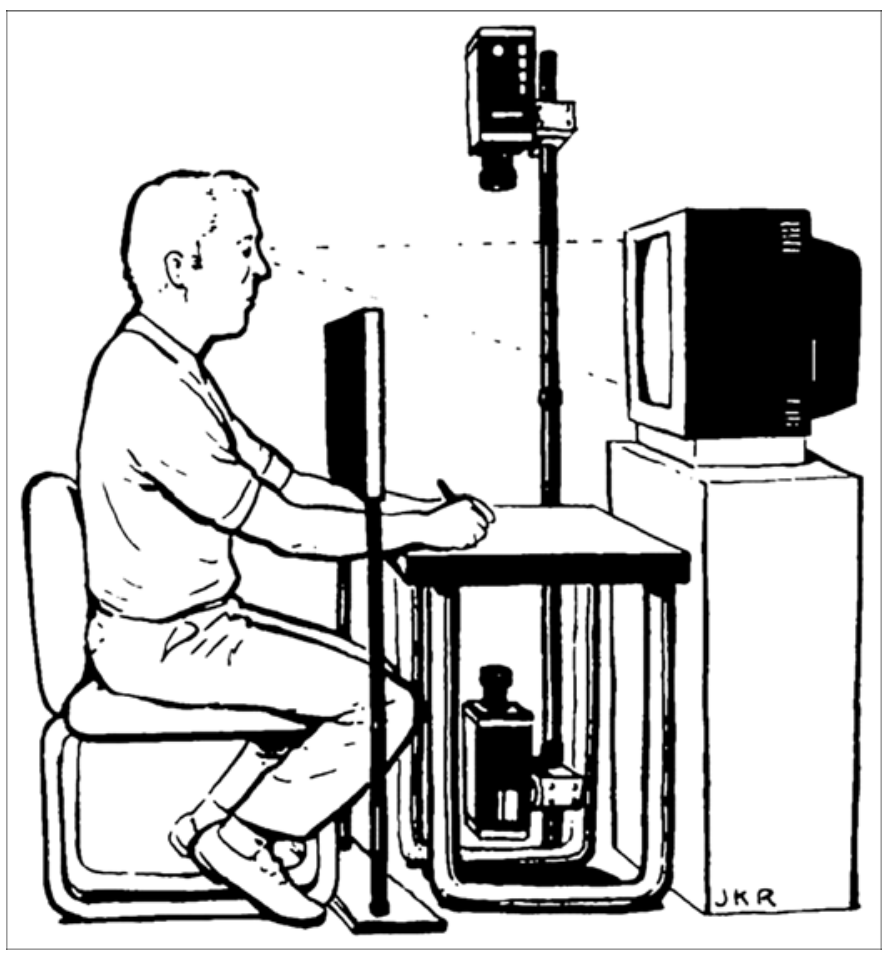

Figure 1.

Video apparatus used in current study (based on Na DL, Adair JC, Williamson DJ, Schwartz RL, Haws B, Heilman KM. Dissociation of sensory-attentional from motor-intentional neglect. J Neurol Neurosurg Psychiatry. 1998;64(3):331-38 [PMID: 9527144]). Subject sees work space via video monitor rather than looking directly at his or her hand and line to be bisected. Camera above work space displays work space for conventional right-left congruent condition (right work space appears on right side of screen, rightward movement appears rightward), and camera below displays work space for right-left reversed condition (right work space appears on left side of screen, rightward movement appears leftward). Component of horizontal bias that reverses direction with reversed feedback is called perceptualattentional; component of horizontal bias that does not reverse direction with reversed feedback is called motor-intentional.

and MI and PA can be calculated from mean linebisection errors in the conventional and RL-reversed conditions. Individual subject's MI and PA biases can be calculated from these data. Each subject's mean for the conventional and RL-reversed conditions can also be used for calculating group MI and PA bias components.

\section{Research Question 1: Effect of Monocular Patching on Line-Bisection Under Conventional Conditions}

Subjects performed line bisections in the following order: (1) unpatched, (2) wearing a right eye patch, and (3) wearing a left eye patch. We first tested all subjects unpatched to confirm that they could participate in the experiment. In both the conventional and RL-reversed conditions, all subjects performed three testing blocks (unpatched, right eye patch, and left eye patch) of 12 line bisections each, except one subject who reported fatigue during testing and completed 6 line bisections each testing block. We sought to understand whether subjects had consistent bias and whether any such bias changed with patching. To address this a priori question, mean linebisection error was calculated and compared with perfect performance for each condition for the group as a whole with one-sample $t$-tests.

\section{Research Question 2: Perceptual-Attentional Versus Motor-Intentional Effect of Monocular Patching}

In the RL-reversed condition, subjects again performed line bisections in three testing blocks (unpatched, right eye patch, and left eye patch) of 12 line bisections each. We aimed to determine whether patching would primarily affect PA or MI spatial bias. To address this a priori question, we calculated mean error for each patching condition for the group as a whole and compared performance in the RL-reversed condition with that obtained during the conventional condition, as described. We then compared PA and MI bias components for the unpatched, right eye patch, and left eye patch conditions in poststroke subjects versus controls with repeated-measures analysis of variance.

\section{Research Question 3: Classification of Individual Subjects as Having Primary Perceptual-Attentional Versus Motor-Intentional Bias}

Group data might not give us information about what happens to individual subject spatial bias. Group analysis for a small, heterogeneous group also presents inherent problems for generalizing results. For that reason, we included analyses of individual subject performance. If a subject's errors were unchanged in the RL-reversed condition, we hypothesized that spatial bias was primarily due to an MI deficit. If a subject's errors changed direction (e.g., from right of center to left of center) in the RLreversed condition and a paired-sample $t$-test revealed that this mean change in performance was significant, we hypothesized that the deficit was primarily PA, or feedback-dependent.

We compared each subject's performance with a right eye patch with his or her performance unpatched (in the conventional condition) to qualitatively examine whether poststroke subjects with one primary bias type 
demonstrate a greater effect of eye patching. We then repeated this comparison for each subject's performance with a left eye patch versus unpatched. All statistical results are reported for two-tailed comparisons.

\section{RESULTS}

\section{Research Question 1: Effect of Monocular Patching on Line-Bisection Under Conventional Conditions}

Data are presented as mean \pm standard deviation. As a group, poststroke subjects erred $3.7 \pm 11.5 \mathrm{~mm}$ rightward while they bisected lines under the conventional condition. This bias, although rightward, was not significantly different from perfect performance $(p=0.47)$ or the performance of controls $(p=0.68)$. These comparisons may not have reached significance because of the small number of subjects rather than an effect size. However, poststroke subjects did not demonstrate significant spatial bias possibly because they no longer had spatial neglect (i.e., they had recovered). However, whether this explanation accounts for the failure to find significant spatial bias is unclear because all six poststroke subjects had significant spatial bias when their individual linebisection errors were compared with perfect performance or when other tasks were considered.

While wearing a right eye patch, poststroke subjects erred $6.7 \pm 10.2 \mathrm{~mm}$ rightward, although this was not significantly inaccurate compared with perfect performance $(p=0.17)$. While wearing a left eye patch, subjects again erred rightward but these errors did not reach statistical significance (11.7 $\pm 17.5 \mathrm{~mm}, p=0.16)$.

\section{Research Question 2: Perceptual-Attentional Versus Motor-Intentional Effect of Monocular Patching}

Results showed a bias type by patch side interaction $(p=0.01$; Figure 2) and a significant bias type by patch side by group interaction $(p=0.03)$.

In the unpatched condition, poststroke subjects' mean rightward bias fractionated into a leftward PA bias ( $-7.6 \pm$ $13.2 \mathrm{~mm})$ and a rightward MI bias $(11.3 \pm 16.8 \mathrm{~mm})$. Across the poststroke group, PA leftward line-bisection error was significantly reduced with either right or left eye patching $(-0.3 \pm 14.1 \mathrm{~mm}$ left eye patch, $-2.3 \pm 14.5 \mathrm{~mm}$ right eye patch, $p<0.05$ for both comparisons) but a patching effect on the group rightward MI bias was not significant (12.0 $\pm 17.6 \mathrm{~mm}$ left eye patch, $p=0.83$; $9.0 \pm$ $18.0 \mathrm{~mm}$ right eye patch, $p=0.08$ ).

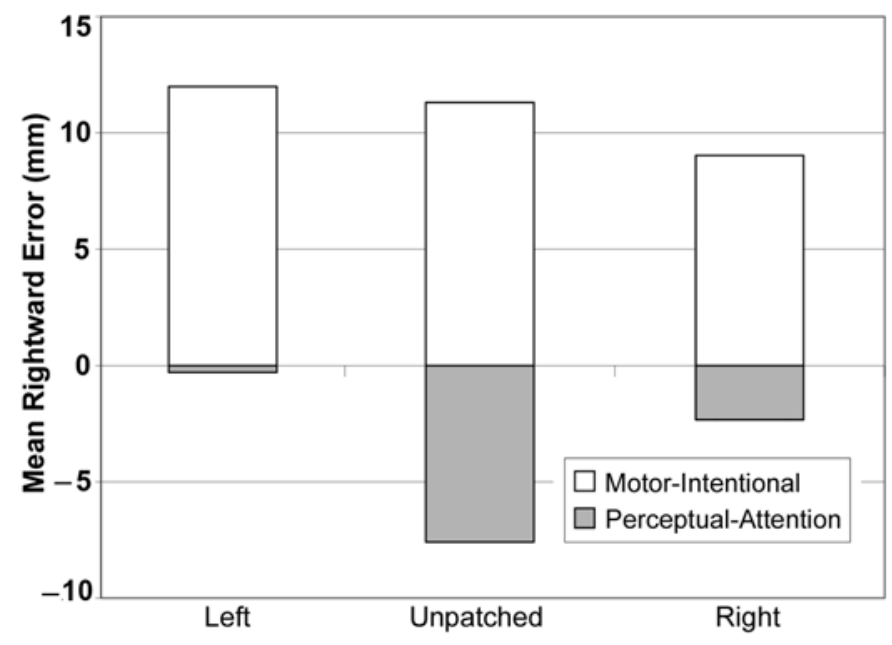

Figure 2.

Poststroke subjects' $(n=6)$ line-bisection performance under left eye patch, unpatched, and right eye patch conditions. With left and right eye patching, change in rightward motor-intentional bias was smaller in magnitude than change in perceptual-attentional (PA) bias (patch side $\times$ bias type interaction, $p=0.01$ ). Both left and right eye patching reduced magnitude of leftward PA bias. No effect of left or right eye patching was demonstrated in control subjects $(n=8)$.

No significant alterations in PA or MI bias with the right or left eye patch were observed in the control group ( $p>0.25$ for all comparisons). These results support a specific effect of eye patching on PA bias in the poststroke group that is distinct from the controls.

\section{Research Question 3: Classification of Individual Subjects as Having Primary Perceptual-Attentional Versus Motor-Intentional Bias}

Compared with perfect performance, five of the six poststroke subjects made significant line-bisection errors in the unpatched conventional condition. In the sixth subject who performed only six trials because of test fatigue, rightward errors may not have reached significance because of insufficient power ( $p=0.13$ ). In support of this subject's abnormal spatial bias (described in the "Methods" section), we observed abnormal, right-biased performance on a line-cancelation task [22].

Of the six poststroke subjects, four made mean rightward line-bisection errors and two made mean leftward line-bisection errors. When line-bisection performance unpatched was compared between the conventional and RL-reversed conditions, two poststroke subjects' errors were classified as consistent with a primary PA bias (i.e., error direction reversed with RL reversal). Four poststroke 
subjects' errors were classified as consistent with a primary MI bias.

We performed pairwise comparisons (Student $t$-test) for the unpatched, left eye patch, and right eye patch conventional conditions to determine whether individual subjects improved with eye patching. Improvement was defined as two-tailed significance $(p<0.05)$ when the unpatched condition was compared with either the left or right eye-patch condition. Of the six poststroke subjects, three showed improved performance with monocular patching (one ipsilesional, one contralesional, one both), two showed worse performance with eye patching (one contralesional, one both), and one showed no significant eye-patching effect. Information by subject is summarized in Table 2.

\section{DISCUSSION}

Because eye patching is commonly used for ocular conditions in the rehabilitation setting and may be an inexpensive treatment for spatial neglect, we wished to understand whether right-hemisphere stroke patients with spatial bias on a line-bisection task would show altered performance with eye patching. We also wished to examine whether eye patching worsened line-bisection performance, which would indicate a need for further studies on the possibly adverse functional impact of this intervention. These preliminary results suggest that monocular patching affects performance on at least one spatial task and that this effect may differ among patients.

Our poststroke subjects cannot be said to have spatial neglect (pathological spatial bias) by conventional criteria. All the poststroke subjects except one had significant spatial bias on the line-bisection task compared with perfect performance, and the subject without spatial bias performed abnormally on a line-cancelation array by omitting left-sided targets. However, no subject performed within the range reported as consistent with spatial neglect on the BIT by the authors of this instrument. Although none of our subjects was living independently at the time of testing, we unfortunately did not collect detailed data about their self-care competence so that we could delineate their disability or determine whether asymmetric performance on daily living tasks occurred. However, all subjects included in the current study met the definition of spatial neglect that we suggested earlier (i.e., spatial bias in a subject with a brain lesion, associated with functional disability).

Two poststroke subjects in the current study (in particular, subject 4) may have demonstrated ipsilesional neglect [23]. They demonstrated leftward line-bisection errors. If indeed they had ipsilesional neglect, they may have demonstrated not only pathological leftward bias but also right-sided bias on other tasks. We indeed observed that subject 6 had rightward bias when canceling lines in an array. However, only a larger study of subjects in the chronic phase of stroke recovery will delineate whether leftward bias on the line-bisection task represents a pathological subtype or a common behavior in the natural history of spatial bias. Whether subjects have primarily contralesional or ipsilesional neglect, or even if subjects have both bias types, we suggest that the current paradigm may be useful in examining alterations of spatial bias with an intervention [24].

Both poststroke subjects with leftward bias had contralesional visual field defects. Although subjects with hemianopia but not spatial neglect may allocate more attention to the contralesional blind field [25], the clinical context suggests that both subjects' leftward errors were pathological. Subject 6 exhibited contralesional neglect on a line-cancelation task (as described in the previous paragraph) and subject 4 had a clinical history of "left-sided grasp on line bisection" despite a noted right gaze preference in the acute phase of stroke recovery. PA bias in the group as a whole was leftward on the line-bisection task. We suggest that leftward bias may occur in the chronic phase of recovery from spatial neglect and may be pathological. However, further research is needed on the effects of monocular patching on subjects with and without hemianopia and with and without spatial neglect.

We cannot ensure that practice or fatigue effects did not confound our results. Further research may need to specifically examine whether subjects improve while performing the line-bisection task or whether significant fatigue alters results in a paradigm that is comparable with ours.

Despite questions about whether our poststroke subject group is comparable with patients with spatial neglect, our results suggest that monocular patching affected bias on the line-bisection task. This effect appeared to be specific to PA bias (i.e., feedbackdependent); eye patching did not affect MI bias across the group of subjects. 


\section{CONCLUSIONS}

An individual patient may demonstrate both PA and MI spatial biases while performing a task. However, one bias type may predominate over a specific task or many tasks. For line-bisection performance, we analyzed whether a primary PA or MI spatial bias accounted for each poststroke subject's performance. Poststroke subjects with both bias types were included in the study. Although the eye-patching effect may have been PA in character across the group of subjects, improved linebisection performance with eye patching did not occur selectively in subjects with a primary PA spatial bias. Some subjects with a primary MI spatial bias also showed improvement (Table 2).

This preliminary study suggests that further data are needed on the effect of monocular patching in larger groups of well-characterized subjects after righthemisphere stroke. However, inclusion of functional tasks in the outcomes to be investigated is also important for determining whether altered performance on spatial tasks with patching is important pragmatically. If alterations that potentially affect daily function occur, the onset and duration of the patching effect also require further study.

\section{ACKNOWLEDGMENTS}

We thank the study participants for donating their time to take part in this research. We are also grateful for the suggestions of three anonymous reviewers on an initial version of this article.

Preliminary results were previously presented to the American Society of Neurorehabilitation and the American Congress of Rehabilitation Medicine. (Barrett AM, Burkholder S. Monocular patching and spatial bias after right hemisphere stroke [abstract]. Neurorehabil Neural Repair. 2002;16(4):360.)

This material was based on work supported by the National Institutes of Health (NIH)/National Institute of Neurological Disorders and Stroke, grant K08 NS002085; the Departments of Medicine and Neurology, The Pennsylvania State University College of Medicine; the Kessler Medical Rehabilitation Research and Education Corporation; and the General Clinical Research Center of The Pennsylvania State University College of Medicine, grants NIH/National Center for Research Resources C06 RR016499 and M01 RR010732.
Dr. Barrett received medication/pharmacy fees from Pfizer/Eisai for an unrelated study and received three lecture honoraria in the last 10 years (Pfizer, Novartis, total amount $<\$ 10,000)$.

\section{REFERENCES}

1. Heilman KM, Watson RT, Valenstein E. Neglect and related disorders. In: Heilman KM, Valenstein E, editors. Clinical neuropsychology. 1st ed. New York (NY): Oxford University Press; 1979. p. 296-346.

2. Mark VW. Acute versus chronic functional aspects of unilateral spatial neglect. Front Biosci. 2003;8:e172-89. [PMID: 12456357]

3. Bowen A, Lincoln NB, Dewey M. Cognitive rehabilitation for spatial neglect following stroke. Cochrane Database Syst Rev. 2002;(2):CD003586. [PMID: 12076489]

4. Na DL, Adair JC, Williamson DJ, Schwartz RL, Haws B, Heilman KM. Dissociation of sensory-attentional from motor-intentional neglect. J Neurol Neurosurg Psychiatry. 1998;64(3):331-38. [PMID: 9527144]

5. Grujic Z, Mapstone M, Gitelman DR, Johnson N, Weintraub S, Hays A, Kwasnica C, Harvey R, Mesulam MM. Dopamine agonists reorient visual exploration away from the neglected hemispace. Neurology. 1998;51(5):1395-98. [PMID: 9818867]

6. Sprague JM. Interaction of cortex and superior colliculus in mediation of visually guided behavior in the cat. Science. 1966;153(743):1544-47. [PMID: 5917786]

7. Posner MI, Rafal RD. Cognitive theories of attention and the rehabilitation of attentional deficits. In: MJ Meir, Benton AL, Diller L, editors. Neuropsychological rehabilitation. New York (NY): Guilford Press; 1987. p. 182201.

8. Taub E, Miller NE, Novack TA, Cook EW 3rd, Fleming WC, Nepomuceno CS, Connell JS, Crago JE. Technique to improve chronic motor deficit after stroke. Arch Phys Med Rehabil. 1993;74(4):347-54. [PMID: 8466415$]$

9. Beis JM, Andre JM, Baumgarten A, Challier B. Eye patching in unilateral spatial neglect: efficacy of two methods. Arch Phys Med Rehabil. 1999;80(1):71-76.

[PMID: 9915375]

10. Butter CM, Kirsch NL. Combined and separate effects of eye patching and visual stimulation on unilateral neglect following stroke. Arch Phys Med Rehabil. 1992;73(12): 1133-39. [PMID: 1463376$]$

11. Soroker N, Cohen T, Baratz C, Glicksohn J. Is there a place for ipsilesional eye patching in neglect rehabilitation? Behav Neurol. 1994;7:159-64. 
12. Serfaty C, Soroker N, Glicksohn J, Sepkuti J, Myslobodsky MS. Does monocular viewing improve target detection in hemispatial neglect? Rest Neurol Neurosci. 1995;9:77-83.

13. Walker R, Young AW, Lincoln NB. Eye patching and the rehabilitation of visual neglect. Neuropsychol Rehabil. 1996;6(3):219-32.

14. Barrett AM, Crucian GP, Beversdorf DQ, Heilman KM. Monocular patching may worsen sensory-attentional neglect: a case report. Arch Phys Med Rehabil. 2001;82(4): 516-18. [PMID: 11295013]

15. Barrett AM, Crucian GP, Heilman KM. Eye patching biases spatial attention after thalamic hemorrhage in a patient without spatial neglect: a case study. Arch Phys Med Rehabil. 2004;85(6):1017-20. [PMID: 15179660]

16. Wallace SF, Rosenquist AC, Sprague JM. Ibotenic acid lesions of the lateral substantia nigra restore visual orientation behavior in the hemianopic cat. J Comp Neurol. 1990; 296(2):222-52. [PMID: 2358533]

17. Moster ML, Volpe NJ, Kresloff MS. Neuro-opthalmologic findings in closed head injury [abstract]. Neurology. 1999; 52(Suppl 2):A23.

18. Barrett AM, Longin EA, Heilman KM. Monocular patching affects inattention but not perseveration in spatial neglect [abstract]. J Int Neuropsychol Soc. 2002;8(1):317.
19. Adair JC, Na DL, Schwartz RL, Heilman KM. Analysis of primary and secondary influences on spatial neglect. Brain Cogn. 1998;37(3):351-67. [PMID: 9733554]

20. Harvey M, Kramer-McCaffery T, Dow L, Murphy PJ, Gilchrist ID. Categorisation of 'perceptual' and 'premotor' neglect patients across different tasks: is there strong evidence for a dichotomy? Neuropsychologia. 2002;40(8): 1387-95. [PMID: 11931943]

21. Wilson BA, Cockburn J, Halligan P. Behavioural Inattention Test. London (England): Harcourt Assessment; 1987.

22. Albert ML. A simple test of visual neglect. Neurology. 1973;23(6):658-64. [PMID: 4736313]

23. Kim M, Na DL, Kim GM, Adair JC, Lee KH, Heilman KM. Ipsilesional neglect: behavioural and anatomical features. J Neurol Neurosurg Psychiatry. 1999;67(1):35-38. [PMID: 10369819]

24. Schwartz RL, Barrett AM, Kim M, Heilman KM. Ipsilesional intentional neglect and the effect of cueing. Neurology. 1999;53(9):2017-22. [PMID: 10599774]

25. Barton JJ, Black SE. Line bisection in hemianopia. J Neurol Neurosurg Psychiatry. 1998;64(5):660-62.

[PMID: 9598685]

Submitted for publication January 14, 2005. Accepted in revised form January 9, 2006. 
